\title{
Development of a photophoretic optical guide for femtosecond $x$-ray diffractive imaging of aerosolized nanoparticles (Conference Presentation)
}

\author{
Richard A. Kirian, Arizona State Univ. (United States); Salah Awel, Ctr. for Free-Electron \\ Laser Science, Deutsches Elektronen-Synchrotron (Germany); Max Wiedorn, Daniel Horke, \\ Nils Roth, Ctr. for Free-Electron Laser Science (Germany); Niko O. Eckerskorn, The \\ Australian National Univ. (Australia); Jochen Küpper, Ctr. for Free-Electron Laser Science, \\ Deutsches Elektronen-Synchrotron (Germany); Henry N. Chapman, Ctr. for Free-Electron \\ Laser Science (Germany); Andrei V. Rode, The Australian National Univ. (Australia)
}

\begin{abstract}
Optical trapping of light-absorbing particles in a gas environment is usually dominated by laser-induced thermal or photophoretic forces, which can be orders of magnitude higher than the force due to radiation pressure. Particle guiding with photophoretic forces over large distances in open air was recently realised by an optical pipeline, formed by a vortex laser beam of doughnut-like intensity profile, with a high-intensity ring of light that surrounds a dark core. We are adapting the optical pipeline concept for the purpose of guiding aerosolized particles into the intense focus of a x-ray free-electron laser (XFEL), in order to enable high-efficiency femtosecond x-ray coherent diffractive imaging (CDI). XFEL-based CDI allows single-shot nanometer-resolution imaging, and multi-shot Angstrom-resolution tomography in the case of reproducible nanoparticles, at a time resolution better than 10 femtoseconds. Remarkably, by imaging at timescales shorter than atomic motion, the crucial resolution-limiting effects of radiation damage may be overcome for radiation-sensitive targets such as viruses and biomolecules. Following on our previous work, we are developing an optical first-order Bessel-like beam with a variable-diameter hollow core and an axial-to-lateral aspect ratio up to $\sim 2000$, that can be used to guide particles with a spatial precision of less than a few $\mu \mathrm{m}$ over centimetre-long distances. We present the ways to control the beam divergence aiming to focus the stream of particles by thermal forces and forces of radiation pressure, analyse the forces acting on the particle in the beam, and uncover the beam structure and intensity to apply for a real-time experiment with XFEL.
\end{abstract}

View presentation recording on the SPIE Digital Library: http://dx.doi.org/10.1117/12.2239171.5161456718001

Optical Trapping and Optical Micromanipulation XIII, edited by Kishan Dholakia, Gabriel C. Spalding, Proc. of SPIE Vol. 9922, 99220K · (C) 2016 SPIE · CCC code: 0277-786X/16/\$18 · doi: 10.1117/12.2239171 